\title{
Asequibilidad de cerveza y bebidas azucaradas para 15 países de América Latina
}

\author{
Guillermo Paraje ${ }^{1}$ y Pablo Pincheira ${ }^{1}$
}

Forma de citar Paraje G, Pincheira P. Asequibilidad de cerveza y bebidas azucaradas en 15 países de América Latina. Rev Panam Salud Publica. 2018;42:e49. https:/ /doi.org/10.26633/RPSP.2018.49

RESUMEN Objetivos. El objetivo de este trabajo fue analizar la evolución de la asequibilidad de la cerveza y de las gaseosas para quince países de América Latina.

Métodos. Los datos corresponden a estadísticas oficiales de índices de precios de cerveza y gaseosas/refrescos, indice de precios al consumidor e indice de salarios nominales en frecuencia mensual. El método se basa en realizar un análisis econométrico, mediante series temporales, para medir el valor esperado de la tasa de crecimiento del indicador de asequibilidad absoluta (utilizando salarios nominales) o relativa (usando precios generales) de las gaseosas y de las cervezas. Resultados. En nueve de los quince países analizados, la asequibilidad (absoluta o relativa), ya sea de bebidas azucaradas o cervezas, ha aumentado de forma estadísticamente significativa. En el caso de las bebidas azucaradas, la asequibilidad absoluta aumentó en cinco países (Chile, Colombia, Costa Rica, Ecuador y Uruguay) y disminuyó en México. En el caso de la cerveza, aumentó en Colombia y Ecuador, se mantuvo inalterada en Argentina, Brasil, Chile, Costa Rica y Uruguay, y se redujo en México.

Conclusiones. A pesar de que la mayoría de los países poseen impuestos a la cerveza y a las bebidas azucaradas, su efecto en los precios no ha sido suficiente para reducir su asequibilidad en todos los países de la muestra. Los impuestos deberían modificarse para reducir la asequibilidad de estas bebidas y afectar su consumo.

Palabras clave Economía de la salud; bebidas alcohólicas; bebidas gaseosas; América Latina.

Los impactos negativos sobre la salud del consumo de bebidas alcohólicas y de bebidas azucaradas han sido establecidos fehacientemente en la bibliografía. El consumo de alcohol está asociado con trastornos físicos relacionados con el daño a diversos órganos (1-3), trastornos psicológicos, asociados con el consumo abusivo y el comportamiento violento (4), accidentes de tránsito (5), embarazos

\footnotetext{
Escuela de Negocios, Universidad Adolfo Ibáñez, Santiago de Chile, Chile. La correspondencia se debe dirigir a Guillermo Paraje. guillermo. paraje@uai.cl
}

no deseados, enfermedades de transmisión sexual, etc. $(6,7)$. Estimaciones recientes muestran que el consumo de alcohol puro por adulto en la Región de las Américas es superior al del promedio mundial, lo que conlleva una alta carga de enfermedad: $4,7 \%$ de las muertes y 6,7\% de los Años de Vida Saludable Perdidos (AVISA) pueden atribuirse directamente a dicho consumo (8).

En el caso de las bebidas azucaradas, la situación es similar. Su consumo está directamente ligado a un incremento del peso corporal y de la ingesta de calorías, incluso por encima de los niveles aportados por dichas bebidas (por efectos de menor saciedad) (9). Además, está asociado directamente con muertes y AVISA producidos por enfermedades cardiovasculares, cánceres y diabetes. El porcentaje de AVISA directamente asociados con el consumo de bebidas azucaradas en América Latina (1,4\% del total de AVISA) duplica el porcentaje mundial y es más alto que el de cualquier otra región (10).

El consumo total de cerveza (la bebida alcohólica más consumida en la Región) y de bebidas azucaradas carbonatadas

Este es un artículo de acceso abierto distribuido bajo los términos de la licencia Creative Commons Attribution-NonCommercial-NoDerivs 3.0 IGO, que permite su uso, distribución y reproducción en cualquier medio, siempre que el trabajo original se cite de la manera adecuada. No se permiten modificaciones a los artículos ni su uso comercial. Al reproducir un artículo no debe haber ningún indicio de que la OPS o el artículo avalan a una organizaciố un producto específico. El uso del logo de la OPS no está permitido. Esta leyenda debe conservarse, junto con la URL original del artículo. 
(las bebidas azucaradas de mayor consumo en la Región) ha crecido en términos per cápita. Entre 2002 y 2016, por ejemplo, el consumo total de cervezas en trece países de la Región (Argentina, Bolivia, Brasil, Chile, Colombia, Costa Rica, Ecuador, Guatemala, México, Perú, República Dominicana, Uruguay y Venezuela) creció a una tasa anual promedio de 2,9\%, muy por encima de la tasa anual promedio de crecimiento poblacional $(1,2 \%)(11,12)$. Sólo en el caso de Venezuela el crecimiento anual promedio del consumo de cerveza fue negativo. Para el resto de los países aumentó desde 1,7\% (Argentina) hasta 10,4\% (Guatemala).

Respecto a las bebidas gaseosas, el consumo anual promedio de estos trece países entre 2002 y 2016 creció a una tasa de $1,7 \%$. Sólo Venezuela tuvo un crecimiento anual promedio negativo y, entre el resto de países, Ecuador tuvo el menor crecimiento anual promedio $(0,03 \%)$, mientras que Uruguay tuvo el mayor crecimiento $(9 \%)(11)$.

Tal como sucede con todos los bienes, dos variables clave para explicar el consumo de estas bebidas son su precio relativo y el ingreso real de los consumidores. Ceteris paribus, un precio relativo mayor de estas bebidas debiera desalentar su consumo, mientras que, si se trata de bienes normales (inferiores), un mayor ingreso real de los consumidores debiera aumentar (disminuir) la demanda por ellas. Si el ingreso de los consumidores se incrementa (disminuye) proporcionalmente más que el precio de las bebidas, aumenta (disminuye) su asequibilidad (por ejemplo, se necesitan menos (más) minutos de trabajo para comprar una unidad de ellas) y, de acuerdo con la evidencia existente para el alcohol $(13,14)$ y el tabaco $(15)$, aumenta (disminuye) su demanda. No se conocen estudios que hayan medido este efecto sobre el consumo de bebidas azucaradas, pero parece razonable suponer que esta relación se encuentra también en este grupo de bienes.

La magnitud del cambio relativo en el consumo resultante de cambios en precios y de los ingresos dependerá de las elasticidades-precio e ingreso de la demanda. Los pocos estudios realizados en América Latina muestran que las bebidas alcohólicas son inelásticas (aunque la elasticidad-precio depende de la bebida alcohólica) y las bebidas azucaradas son elásticas (16-21). Se conoce menos aún sobre la elasticidad-ingreso, aunque la evidencia que existe en la Región apunta a que ambos tipos de bebidas son bienes normales $(16,21)$. Esta evidencia señalaría, entre otras cosas, que impuestos aplicados sobre estas bebidas tendrían un impacto en su consumo, que sería más importante en el caso de las bebidas azucaradas $(22,23)$.

Un número importante de países de América Latina grava con impuestos las bebidas alcohólicas y azucaradas, pero no se conoce si dichos impuestos han sido efectivos para disminuir la asequibilidad de estos bienes. El objetivo principal de este estudio fue analizar, mediante la realización de un análisis econométrico de series temporales, la evolución de la asequibilidad de la cerveza (como proxy de las bebidas alcohólicas) y de las gaseosas (como proxy de las bebidas azucaradas) para un conjunto de países de la Región. Según Euromonitor Internacional, el consumo de cerveza representa, en promedio para los 15 países de la Región para los que recopilan datos, $85 \%$ del consumo total de bebidas alcohólicas, mientras que el de gaseosas representa $77 \%$ del total de bebidas azucaradas (11).

\section{Materiales y métodos}

Los datos recopilados corresponden a estadísticas oficiales de índices de precios de cerveza y gaseosas/refrescos, del índice de precios generales al consumidor (IPC) y del índice de salarios nominales, en 15 países de América Latina. En este estudio se analizó la situación tributaria de bebidas alcohólicas y azucaradas en 15 países: Argentina, Bolivia, Brasil, Chile, Colombia, Costa Rica, Ecuador, El Salvador, Guatemala, Honduras, México, Paraguay, Perú, República Dominicana y Uruguay. De ellos, todos gravan con impuestos las bebidas alcohólicas y en sólo dos (Colombia y República Dominicana) las bebidas azucaradas no están gravadas. Las series, todas expresadas en frecuencia mensual, proceden de los Institutos de Estadística nacionales o de los Bancos Centrales, según se consigna en el cuadro 1.

En la mayoría de los países, los precios de la cerveza y las gaseosas corresponden al índice de estos productos que se incluye en el IPC. En Argentina se usa dicho componente, pero del Índice de Precios Industriales Mayoristas (IPIM) debido a las serias dudas sobre la medición del IPC desde 2007 (24). No se tienen dudas al respecto en el caso del IPIM. En Perú se usan precios nominales recopilados por el Instituto Nacional de Estadística e Informática en Lima Metropolitana.

En cuanto a los salarios, sólo se encontró información mensual para ocho países. En la mayoría de los casos corresponden a índices de salarios nominales generales, a excepción de Costa Rica, país para el cual se obtuvo un índice de salarios mínimos. En Colombia existe un índice de salarios medios en la industria manufacturera para dos tipos de empleos (obreros y empleados) con datos hasta diciembre de 2014 y otro para el total de la industria manufacturera con datos desde enero de 2014. En este estudio se usó un promedio simple de los dos índices disponibles hasta diciembre de 2014 y a partir de allí se lo empalmó con el del total de la industria. El cuadro 2 muestra las estadísticas descriptivas de los datos usados, entre los cuales se incluye el número de observaciones de cada índice por país, su media, desviación estándar y mediana.

El método utilizado se basa en medir el valor esperado de la tasa de crecimiento del indicador de asequibilidad de las gaseosas y de las cervezas en cada uno de los quince países de la muestra. Para un país específico, esta tasa de crecimiento se define como:

$$
\gamma_{t}^{A}=100 *\left[\frac{R_{t}-R_{t-j}}{R_{t-j}}\right]=100 *\left[\frac{R_{t}}{R_{t-j}}-1\right]
$$

donde

$$
\mathrm{R}_{\mathrm{t}}=\frac{W_{t}}{P_{t}}
$$

denota el indicador de asequibilidad mensual del bien en cuestión, definido como el cociente entre el salario nominal $\left(\mathrm{W}_{\mathrm{t}}\right)$ y el precio del bien $\left(\mathrm{P}_{\mathrm{t}}\right)$. En ausencia de datos de remuneraciones, el indicador de asequibilidad se construye como el cociente entre el Índice de Precios al Consumidor y el precio del bien. Cuando este sea el caso, este indicador se considera como uno de asequibilidad relativa. El entero $\mathrm{j}$ en nuestro caso puede tomar los valores $\mathrm{j}=1, \mathrm{j}=6 \mathrm{o} \mathrm{j}=12$. En el primer caso, se analiza la tasa de crecimiento mensual de la asequibilidad, en el segundo, la semestral y en el tercero, la tasa de crecimiento anual. La elección de un valor de j para cada país y bebida depende de dos razones técnicas: 
CUADRO 1. Fuentes y tipo de datos utilizados para el estudio por país, variable y periodo abarcado

\begin{tabular}{|c|c|c|c|c|}
\hline País & Variable & Datos utilizados & Inicio-Final & Fuente \\
\hline \multirow[t]{3}{*}{ Argentina } & Cerveza & Índice de Precios Internos al por Mayor & oct 2001-oct 2015 & INDEC \\
\hline & General & Índice de Precios Internos al por Mayor & oct 2001-oct 2015 & INDEC \\
\hline & Salarios & Índice de Salarios Medio Nominal & oct 2001-oct 2015 & INDEC \\
\hline Bolivia & General & Índice de Precios al Consumidor & mar 2008-may 2016 & INE \\
\hline \multirow[t]{2}{*}{ Brasil } & Cerveza & Índice Nacional de Precios al Consumidor & mar 2002-feb 2016 & SIDRA \\
\hline & General & Índice Nacional de Precios al Consumidor & mar 2002-feb 2016 & SIDRA \\
\hline \multirow[t]{4}{*}{ Chile } & Cerveza & Índice de Precios al Consumidor & ene 2009-may 2016 & INE \\
\hline & Bebidas gaseosas & Índice de Precios al Consumidor & ene 2009-may 2016 & INE \\
\hline & General & Índice de Precios al Consumidor & ene 2009-may 2016 & INE \\
\hline & Salarios & Índice Nominal de Remuneraciones & ene 2009-may 2016 & INE \\
\hline \multirow[t]{2}{*}{ Colombia } & Cerveza & Índice de Precios al Consumidor & ene 2009-abr 2016 & DANE \\
\hline & Gaseosas y maltas & Índice de Precios al Consumidor & ene 2009-abr 2016 & DANE \\
\hline \multirow{3}{*}{ Costa Rica } & Bebidas gaseosas & Índice de Precios al Consumidor & jul 2006-jun 2016 & INEC \\
\hline & General & Índice de Precios al Consumidor & jul 2006-jun 2016 & INEC \\
\hline & Salarios & Índice de salarios mínimos nominales & jul 2006-jun 2016 & INEC \\
\hline \multirow[t]{4}{*}{ Ecuador } & Cerveza & Índice de Precios al Consumidor & ene 2005-jun 2016 & INEC \\
\hline & Gaseosas & Índice de Precios al Consumidor & ene 2005-jun 2016 & INEC \\
\hline & General & Índice de Precios al Consumidor & ene 2005-jun 2016 & INEC \\
\hline & Salarios & Índice General de Remuneraciones & ene 2003-jun 2016 & INEC \\
\hline \multirow[t]{3}{*}{ El Salvador } & Cerveza & Índice de Precios al Consumidor & ene 2006-may 2016 & Banco Central de El Salvador \\
\hline & Gaseosas & Índice de Precios al Consumidor & ene 2006-may 2016 & Banco Central de El Salvador \\
\hline & General & Índice de Precios al Consumidor & ene 2006-may 2016 & Banco Central de El Salvador \\
\hline \multirow{2}{*}{ México } & General & Índice de Precios al Consumidor & ene 2007-mar 2016 & INEGI \\
\hline & Salarios & Índice de Remuneraciones Media por persona ocupada & ene 2007-mar 2016 & Banco de México \\
\hline \multirow[t]{3}{*}{ Paraguay } & Cerveza & Índice de Precios al Consumidor & dic 2007-sep 2016 & Banco Central del Paraguay \\
\hline & Bebidas Gaseosas & Índice de Precios al Consumidor & dic 2007-sep 2016 & Banco Central del Paraguay \\
\hline & General & Índice de Precios al Consumidor & dic 2007-sep 2016 & Banco Central del Paraguay \\
\hline \multirow[t]{3}{*}{ Perú } & Cerveza blanca mediana & Precio nominal para Lima & ene 1996-nov 2016 & INEI \\
\hline & Gaseosas & Precio nominal para Lima & ene 1996-nov 2016 & INEI \\
\hline & General & Índice de Precios al Consumidor para Lima & ene 1996-nov 2016 & INEI \\
\hline \multirow[t]{3}{*}{ R. Dominicana } & Cerveza envasada & Índice de Precios al Consumidor & ene 2011-jun 2016 & ONE \\
\hline & Refrescos & Índice de Precios al Consumidor & ene 2011-jun 2016 & ONE \\
\hline & General & Índice de Precios al Consumidor & ene 2011-jun 2016 & ONE \\
\hline \multirow[t]{4}{*}{ Uruguay } & Cerveza & Índice de Precios al Consumidor & mar 1997-may 2016 & INE \\
\hline & Refrescos & Índice de Precios al Consumidor & mar 1997-may 2016 & INE \\
\hline & General & Índice de Precios al Consumidor & mar 1997-may 2016 & INE \\
\hline & Salarios & Índice Medio de Salarios & mar 1997-may 2016 & INE \\
\hline
\end{tabular}

INDEC: Instituto Nacional de Estadísticas y Censo; INE: Instituto Nacional de Estadística; SIDRA: Sistema IBGE de Recuperação Automática; DANE: Departamento Administrativo Nacional de Estadísticas; INEGI: Instituto Nacional de Estadística y Geografía; INEI: Instituto Nacional de Estadísticas e Informática; ONE: Oficina Nacional de Estadísticas. 
CUADRO 2. Estadísticas descriptivas de los índices de precios y salarios por país

\begin{tabular}{|c|c|c|c|c|c|c|}
\hline País & $\begin{array}{c}\text { Número de } \\
\text { observaciones }\end{array}$ & Media & $\begin{array}{l}\text { Desvíación } \\
\text { estándar }\end{array}$ & Mediana & Mínimo & Máximo \\
\hline \multicolumn{7}{|c|}{ Îndice de precios gaseosas/refrescos } \\
\hline Chile & 91 & 116,2 & 12,1 & 113,4 & 100,0 & 142,1 \\
\hline Colombia & 90 & 120,4 & 10,1 & 124,2 & 100,0 & 136,2 \\
\hline Costa Rica & 125 & 146,6 & 23,9 & 153,5 & 100,0 & 175,6 \\
\hline Ecuador & 138 & 129,4 & 26,5 & 121,6 & 94,4 & 212,2 \\
\hline El Salvador & 125 & 107,1 & 16,4 & 100,7 & 81,9 & 142,4 \\
\hline Guatemala & 63 & 110,1 & 6,1 & 110,9 & 100,0 & 119,9 \\
\hline Honduras & 53 & 111,8 & 7,5 & 115,6 & 100,0 & 125,5 \\
\hline México & 119 & 128,4 & 20,3 & 123,4 & 100,0 & 164,2 \\
\hline Paraguay & 102 & 122,4 & 14,8 & 120,7 & 97,9 & 148,6 \\
\hline Perú & 251 & 91,9 & 10,4 & 92,2 & 73,8 & 113,6 \\
\hline Rep. Dominicana & 66 & 105,7 & 2,3 & 105,4 & 100,0 & 109,3 \\
\hline \multicolumn{6}{|c|}{ Índice de precios cerveza } & 302,5 \\
\hline Argentina & 169 & 371,5 & 138,6 & 369,5 & 98,0 & 665,6 \\
\hline Bolivia & 99 & 161,1 & 34,6 & 166,6 & 100,0 & 208,0 \\
\hline Brasil & 173 & 170,9 & 53,2 & 146,6 & 100,0 & 278,3 \\
\hline Chile & 91 & 113,2 & 13,6 & 108,5 & 97,4 & 143,6 \\
\hline Colombia & 90 & 113,3 & 4,8 & 114,1 & 100,0 & 120,8 \\
\hline Costa Rica & 125 & 156,3 & 30,0 & 159,5 & 100,0 & 193,1 \\
\hline Ecuador & 138 & 126,7 & 22,3 & 123,7 & 99,2 & 185,0 \\
\hline El Salvador & 125 & 110,0 & 6,3 & 112,8 & 99,6 & 119,8 \\
\hline Guatemala & 63 & 97,8 & 5,6 & 95,2 & 92,0 & 106,8 \\
\hline Honduras & 53 & 106,1 & 4,8 & 107,2 & 100,0 & 114,1 \\
\hline México & 119 & 133,4 & 21,1 & 132,4 & 99,4 & 165,7 \\
\hline Paraguay & 102 & 143,3 & 23,3 & 146,6 & 100,0 & 172,7 \\
\hline Perú & 251 & 136,1 & 15,2 & 134,7 & 100,0 & 166,0 \\
\hline Rep. Dominicana & 66 & 121,4 & 16,2 & 121,7 & 100,0 & 146,1 \\
\hline Uruguay & 232 & 257,7 & 120,4 & 229,0 & 100,0 & 537,9 \\
\hline \multicolumn{7}{|c|}{ Índice de precios generales } \\
\hline Argentina & 169 & 403,7 & 202,6 & 348,7 & 98,4 & 914,1 \\
\hline Bolivia & 101 & 128,8 & 16,5 & 128,5 & 100,0 & 157,5 \\
\hline Brasil & 173 & 169,0 & 41,0 & 163,0 & 100,0 & 262,6 \\
\hline Chile & 91 & 108,3 & 7,9 & 107,1 & 97,8 & 129,1 \\
\hline Colombia & 90 & 111,8 & 8,1 & 111,0 & 100,0 & 131,8 \\
\hline Costa Rica & 125 & 144,7 & 22,0 & 147,9 & 100,0 & 171,3 \\
\hline Ecuador & 138 & 128,0 & 18,0 & 127,1 & 100,0 & 157,0 \\
\hline El Salvador & 125 & 118,5 & 8,2 & 119,4 & 100,0 & 127,9 \\
\hline Guatemala & 63 & 110,4 & 5,8 & 110,8 & 100,0 & 120,8 \\
\hline Honduras & 54 & 112,4 & 6,6 & 113,8 & 100,0 & 122,2 \\
\hline México & 119 & 122,7 & 13,5 & 123,4 & 99,9 & 145,4 \\
\hline Paraguay & 102 & 122,2 & 13,2 & 124,5 & 100,0 & 144,9 \\
\hline Perú & 251 & 152,8 & 26,6 & 148,1 & 100,0 & 205,9 \\
\hline Rep. Dominicana & 66 & 112,7 & 5,5 & 114,4 & 100,0 & 119,6 \\
\hline Uruguay & 232 & 244,6 & 106,4 & 224,4 & 100,0 & 485,4 \\
\hline \multicolumn{7}{|l|}{ Índice de salarios } \\
\hline Argentina & 169 & 409,3 & 345,3 & 277,7 & 99,3 & 1423,0 \\
\hline Brasil & 168 & 172,2 & 55,5 & 163,5 & 100,0 & 277,9 \\
\hline Chile & 89 & 120,4 & 14,9 & 119,2 & 100,0 & 147,4 \\
\hline Colombia & 89 & 131,3 & 14,7 & 128,1 & 100,0 & 163,1 \\
\hline Costa Rica & 120 & 153,8 & 31,3 & 155,3 & 100,0 & 198,1 \\
\hline Ecuador & 134 & 217,6 & 83,3 & 204,5 & 100,0 & 360,8 \\
\hline México & 118 & 130,3 & 17,2 & 128,3 & 99,9 & 182,5 \\
\hline Uruguay & 231 & 256,3 & 141,5 & 197,8 & 100,0 & 599,8 \\
\hline
\end{tabular}

la necesidad de eliminar componentes estacionales de las series y la necesidad de trabajar con tasas de asequibilidad estacionarias para evitar resultados espurios. Para eliminar elementos estacionales de carácter aditivo, lo natural es trabajar con tasas anuales de asequibilidad. No obstante, la prueba de raíz unitaria de Phillips-Perron no permite rechazar la hipótesis nula de raíz unitaria en todas las series anuales. Sí permite, en cambio, rechazar la hipótesis de raíz unitaria en todas las series de tasas de asequibilidad mensual $(j=1)$ y en la mayoría de las series semestrales, a niveles de significación estadística habituales. Ante esta situación, optamos por trabajar preferentemente con tasas de asequibilidad anual siempre y cuando con la prueba de Phillips-Perron se rechace la existencia de raíz unitaria para estas series. Cuando no se rechaza, optamos por trabajar con tasas de asequibilidad semestral, también en la medida en que se rechace la existencia de raíz unitaria. Finalmente, si para un determinado país y bebida no es posible rechazar la existencia de raíz unitaria en series anuales ni semestrales, trabajamos en última instancia con tasas de asequibilidad mensuales. Esto ocurre con la República Dominicana en gaseosas y Guatemala y Honduras en cervezas.

Por homogeneidad, en los cuadros se presentan valores anualizados para facilitar la interpretación y comparación entre países. (Los cuadros del análisis de raíz unitaria no se presentan para ahorrar espacio, pero están disponibles de parte de los autores si se solicitan.) Usando una expansión de Taylor de primer orden en torno a $\mathrm{x}=0$, se tiene que la tasa de crecimiento de la asequibilidad se puede aproximar por

$$
\begin{aligned}
\gamma_{t}^{A} & \cong 100 *\left(\ln \left[\frac{R_{t}}{R_{t-j}}\right]\right) \\
& =100 *\left(\ln \left(R_{t}\right)-\ln \left(R_{t-j}\right)\right)
\end{aligned}
$$

Sobre la base de las propiedades del logaritmo natural se verifica que

$$
\begin{aligned}
\gamma_{t}^{A} \cong & 100 *\left(\ln \left(R_{t}\right)-\ln \left(R_{t-j}\right)\right) \\
= & 100 *\left\{\left[\ln \left(W_{t}\right)-\ln \left(W_{t-j}\right)\right]\right. \\
& \left.-\left[\ln \left(P_{t}\right)-\ln \left(P_{t-j}\right)\right]\right\}
\end{aligned}
$$


$\mathrm{O}$, de forma resumida

$\gamma_{t}^{A} \cong \gamma_{t}^{W}-\gamma_{t}^{P}$

donde

$$
\begin{aligned}
& \gamma_{t}^{P}=100 *\left(\left[\ln \left(P_{t}\right)-\ln \left(P_{t-j}\right)\right]\right) \\
& \gamma_{t}^{W}=100 *\left(\left[\ln \left(W_{t}\right)-\ln \left(W_{t-j}\right)\right]\right)
\end{aligned}
$$

En otras palabras, la tasa de crecimiento de asequibilidad puede ser aproximada como la diferencia de las aproximaciones logarítmicas de la tasa de crecimiento de los salarios nominales y del precio de las bebidas.

Tal como se ha señalado anteriormente, lo más usual es estimar la tasa de asequibilidad considerando alguna medida de poder adquisitivo de la población (25, 26). Sin embargo, debido a que no se posee información en frecuencia mensual de salarios o ingresos para todos los países, se considera en estos casos, como índice de asequibilidad, al inverso del precio relativo del bien en cuestión respecto del promedio de los bienes considerados en el cálculo del IPC. En estos casos, si el bien baja su precio relativo en el tiempo, puede considerarse que la asequibilidad relativa ha aumentado. Por otro lado, si el precio relativo se incrementa, se concluye que la asequibilidad relativa ha disminuido.

Bajo el supuesto de estacionariedad para la serie $\gamma_{t}^{A}$ se tiene que su valor esperado no depende del tiempo, por lo cual puede denotase simplemente por $\mu$ :

$$
\mu \equiv E\left(\gamma_{t}^{A}\right)
$$

De este modo, el procedimiento de inferencia estadística se centra en $\mu$. Un valor positivo de este parámetro puede interpretarse como una mayor asequibilidad de la población a la bebida en cuestión. Un parámetro $\mu$ negativo conduce al resultado opuesto: habla de una menor asequibilidad de los bienes a la población. Claramente, un valor nulo del parámetro se interpreta como una asequibilidad que no varía con el tiempo.

Formalmente, el análisis puede centrarse en la siguiente hipótesis nula:

$$
\mathrm{H}_{0}: \mu=0
$$

que se evalúa frente a la alternativa:

$$
\mathrm{H}_{\mathrm{A}}: \mu \neq 0
$$

A diferencia de una muestra i.i.d. (independiente e idénticamente distribuida), los datos de series temporales se caracterizan por presentar un patrón de dependencia, lo que invalida los procedimientos de inferencia que omiten esta realidad. No obstante, si al supuesto de estacionariedad de $\gamma_{t}^{A}$ se le adiciona el de sumabilidad absoluta de sus autocovarianzas, se cumple la siguiente versión del teorema central del límite (27):

$$
\sqrt{T}\left[\frac{1}{T} \sum_{t=1}^{T} \gamma_{t}^{A}-\mu\right] \stackrel{\mathrm{D}}{\rightarrow} N\left(0, V^{*}\right)
$$

donde $V^{*}$ denota a la varianza de largo plazo de $\gamma_{t}^{A}$ y T, el tamaño muestral de la serie temporal. Denotemos con $\sigma(\mathrm{T})$ a un estimador consistente de $V^{*}$. Entonces, bajo la hipótesis nula, se tiene que el siguiente estadístico de tipo t se distribuye asintóticamente normal estándar:

$$
\frac{\left[\frac{1}{T} \sum_{t=1}^{T} \gamma_{t}^{A}\right]}{\sigma(\mathrm{T}) / \sqrt{T}} \stackrel{\mathrm{D}}{\rightarrow} N(0,1)
$$

Los estimadores consistentes de la varianza de largo plazo de una serie temporal abundan en la bibliografía, aunque se reconoce como pionero el estimador propuesto por Newey y West (28). Este estimador requiere la elección de un parámetro de ancho de banda, que en este estudio se escoge de acuerdo con la sugerencia de Newey y West (29).

Una simple manera de construir este estadístico consiste en estimar una regresión entre la variable $\gamma_{t}^{A}$ y una constante, teniendo en cuenta que los errores estándar necesitan un estimador consistente de la varianza de largo plazo, es decir, el estadístico propuesto por Newey y West (28). El método utilizado en el presente estudio incluye una leve modificación de esta estrategia, pues consiste en estimar el siguiente modelo para $\gamma_{t}^{A}$ :

$\gamma_{t}^{A}=\delta+\varepsilon_{t}-\theta \varepsilon_{t-12}$

Donde $\varepsilon_{t}$ es un ruido blanco, con esperanza cero y varianza constante. Del modelo anterior se desprende que

$$
\mu \equiv E\left(\gamma_{t}^{A}\right)=\delta
$$

por lo que la inferencia sobre el parámetro $\mu$ es equivalente a hacer inferencia sobre el intercepto $\delta$ en el modelo aquí usado. La razón por la cual se introduce el término de medias móviles $\theta \varepsilon_{\mathrm{t}-12}$ es para incorporar otra manera de controlar, al menos parcialmente, algunos efectos estacionales que es común encontrar en la series de precios, incluso cuando en ellas se trabaja con tasas anuales (30). De no ser relevante este efecto estacional, se esperaría que el parámetro $\theta$ fuera cercano a cero, y no afectara sustancialmente los resultados de las estimaciones. La estimación de la ecuación (A) se realiza por mínimos cuadrados condicionales, y los errores estándar de los coeficientes se construyen según el estimador mencionado anteriormente propuesto por Newey y West (28).

\section{Resultados}

En el cuadro 3 se presentan los resultados de la estimación de la ecuación (A) para los 12 países para los cuales se dispuso de datos mensuales de precios de bebidas gaseosas. Concretamente, dicho cuadro muestra para cada país la estimación del parámetro $\delta$ en la ecuación $(\mathrm{A})$, su error estándar (en paréntesis) y el correspondiente estadístico de tipo $t$.

Los resultados obtenidos son mixtos. De los seis países para los cuales se midió la asequibilidad considerando los salarios (la manera adecuada de medir asequibilidad), en cinco de ellos (Chile, Colombia, Costa Rica, Ecuador y Uruguay) la hipótesis nula se rechaza con un nivel de significación estadística de $5 \%$, y el coeficiente es positivo, lo que implica un aumento de la asequibilidad de las bebidas gaseosas. Para México, la hipótesis nula también se rechaza con un nivel de significación estadística alto $(1 \%)$, pero el coeficiente es negativo, lo que supone una disminución de la asequibilidad. Tanto Ecuador como Uruguay destacan por presentar los incrementos mayores en las tasas de asequibilidad: 6,37\% anual en Ecuador y $3,54 \%$ en Uruguay.

En el resto de los países no se tienen datos de salarios y, por ende, sólo se tiene un indicador subóptimo de la asequibilidad, que hemos llamado asequibilidad relativa. En estos países se obtienen resultados algo menos concluyentes. En El Salvador, Guatemala y Honduras no puede rechazarse la hipótesis nula de que no hubo cambios en la asequibilidad relativa. En la República Dominicana y Perú, la asequibilidad relativa aumenta en términos estadísticos (se rechaza la hipótesis nula al 10\% y el coeficiente es positivo). En Paraguay, la hipótesis nula se 
rechaza a favor de una disminución de la asequibilidad relativa.

El cuadro 4 es análogo al cuadro 3, pero en él se notifican los resultados de asequibilidad para cervezas en todos los ses considerados para el caso de la cerveza es mayor, aunque también se obtienen resultados mixtos. Para el grupo de países de los cuales se dispone de información sobre salarios y es posible medir mejor la asequibilidad, se tiene que la asequibilidad aumentó significativamente en Colombia y Ecuador. En Colombia lo hizo a una tasa de 4,09\% anual y en Ecuador, de 7,26\%. En Argentina, Brasil, países de la muestra. El número de paí-

Chile, Costa Rica y Uruguay, la tasa de crecimiento de la asequibilidad no es estadísticamente diferente a cero, mientras que en México se habría producido una reducción en la asequibilidad de 1,18\% anual (con un nivel de significación estadística de $10 \%$ ).

Para los países sin series de salarios, es decir, Guatemala y Honduras, se observa un aumento de la asequibilidad relativa estadísticamente significativa a 10\% en dos de ellos. En Perú y El Salvador también se observa un incremento de la asequibilidad relativa, aunque no es estadísticamente significativo. En Bolivia y República Dominicana la asequibilidad

\section{CUADRO 3. Tasa de crecimiento anual desestacionalizada de la asequibilidad en gaseosas por país}

\begin{tabular}{lcccccc}
\hline & Chile & Colombia & Costa Rica & Ecuador & El Salvador & Guatemala \\
\hline Tasa de crecimiento anual & $1,27^{\star * *}$ & $2,75^{* * *}$ & $1,50^{\star * *}$ & $6,37^{\star * *}$ & $-0,57$ & 0,09 \\
Error estándar & $(0,40)$ & $(0,80)$ & 0,31 & $(1,51)$ & $(1,25)$ & $(0,46)$ \\
Estadístico t & 3,17 & 3,43 & 4,81 & 4,23 & 0,45 & 0,19 \\
\hline & Honduras & México & Paraguay & Perú & $\begin{array}{c}\text { República } \\
\text { Dominicana }\end{array}$ & Uruguay \\
\hline Tasa de crecimiento anual & $-0,83$ & $-1,07^{* * *}$ & $-0,49^{*}$ & $2,93^{*}$ & $3,28^{\star *}$ & $3,54^{\star *}$ \\
Error estándar & $(0,53)$ & $(0,21)$ & $(0,26)$ & $(1,76)$ & $(1,66)$ & $(1,65)$ \\
Estadístico t & $-1,55$ & $-5,05$ & 1,92 & 1,66 & 1,97 & 2,14 \\
\hline
\end{tabular}

Notas:

1. Los errores estándar consistentes bajo heterocedasticidad y autocorrelación se han calculado de acuerdo con el método de Newey y West $(28,29)$.

2. Para Chile, Colombia, Costa Rica, Ecuador, México y Uruguay la asequibilidad se mide en relación con el índice de remuneraciones. Para el resto de los países es medida en relación con el Índice de Precios al Consumidor.

3. Para Chile, Colombia, Ecuador y Guatemala, el análisis se basó en la tasa de crecimiento semestral, por lo que la tasa anual notificada es simplemente el doble de la tasa de crecimiento semestral. Para República Dominicana el análisis se basó en la tasa de crecimiento mensual, por lo que la tasa de crecimiento anual notificada es 12 veces la tasa de crecimiento mensual.

4. ${ }^{\star} P<0,10,{ }^{* \star} P<0,05,{ }^{* \star *} P<0,01$. relativa disminuye de forma estadísticamente significativa, como en Paraguay, aunque sin significación estadística.

\section{Discusión}

El aumento del consumo de productos que tengan efectos negativos en la salud de la población es una preocupación de salud pública. La pérdida de años saludables de vida y el incremento de gastos sanitarios asociado con dichos consumos son motivos de inquietud tanto en países desarrollados como en vías de desarro1lo. En la medida en que disminuya la asequibilidad de estos bienes es esperable, dada la evidencia disponible $(13,14)$ que disminuya su consumo. Los resultados de este estudio muestran que, tanto en el caso de las cervezas como de las gaseosas, la tendencia mayoritaria en la Región es hacia un aumento de la asequibilidad o su mantenimiento. En 12 de 15 países la asequibilidad de la cerveza aumentó o se mantuvo inalterada en términos estadísticos, lo cual sucedió también con las gaseosas en 10 de 12 países. Según la evidencia existente (13-15), no sorprende que en la mayoría de los países haya aumentado de manera sostenida el consumo per capita de ambos bienes.

Una política que ha demostrado ser efectiva para disminuir el consumo de este tipo de bienes son los impuestos (3133). Sin embargo, a pesar de que en la gran mayoría de los países de la Región las bebidas azucaradas y alcohólicas están gravadas con impuestos, el presente

\section{CUADRO 4. Tasa de crecimiento anual desestacionalizada de la asequibilidad en cervezas por país}

\begin{tabular}{|c|c|c|c|c|c|}
\hline & Argentina & Bolivia & Brasil & Chile & Colombia \\
\hline Tasa de crecimiento anual & 4,73 & $-4,07^{\star \star \star}$ & 0,10 & 0,65 & $4,09^{\star \star \star}$ \\
\hline Error estándar & $(5,45)$ & $(1,32)$ & $(0,99)$ & $(1,19)$ & $(1,46)$ \\
\hline Estadístico t & 0,87 & 3,08 & 0,10 & 0,55 & 2,80 \\
\hline Tasa de crecimiento anual & 0,53 & $7,26^{\star \star *}$ & 0,50 & $5,26^{* *}$ & $1,42^{*}$ \\
\hline Error estándar & (036) & $(1,50)$ & $(0,62)$ & $(2,64)$ & $(0,80)$ \\
\hline Estadístico t & 1,18 & 4,85 & 0,81 & 2,00 & 1,79 \\
\hline Tasa de crecimiento anual & $-1,18^{\star}$ & $-2,30$ & 1,11 & $-5,04^{\star \star \star}$ & 0,61 \\
\hline Error estándar & $(0,64)$ & $(1,47)$ & $(0,91)$ & 0,40 & $(1,73)$ \\
\hline Estadístico t & $-1,84$ & $-1,57$ & 1,21 & $-12,58$ & 0,32 \\
\hline
\end{tabular}

Notas:

1. Los errores estándar consistentes bajo heterocedasticidad y autocorrelación se han calculado de acuerdo con el método de Newey y West (28, 29).

2. Para Argentina, Brasil, Chile, Colombia, Costa Rica, Ecuador, México y Uruguay, la asequibilidad se mide en relación con el índice de remuneraciones. Para el resto de los países se mide en relación con el Índice de Precios al Consumidor.

3. Para Argentina, Bolivia, Chile, Colombia, Ecuador, El Salvador, Paraguay y Uruguay el análisis se basó en la tasa de crecimiento semestral, de manera que la tasa de crecimiento anual notificada es dos veces la tasa de crecimiento semestral. Para Guatemala y Honduras el análisis se basó en la tasa de crecimiento mensual, por lo que la tasa anual notificada es 12 veces la tasa de crecimiento mensual.

4. ${ }^{*} P<0,10,{ }^{* *} P<0,05,{ }^{* * *} P<0,01$. 
estudio demuestra que la aplicación de estos impuestos ha sido hasta ahora insuficiente para disminuir significativamente su asequibilidad.

Está claro que en ciertos países estos impuestos se han implementado con fines recaudatorios antes que correctivos de las conductas insalubres. Respecto a las bebidas azucaradas, por ejemplo, países como Argentina, Perú o Uruguay gravan estas bebidas así como a sus alternativas saludables, como el agua embotellada. En otros países, como Chile o Ecuador, han aumentado los impuestos de estas bebidas, aunque de manera insuficiente y en el contexto de reformas tributarias motivadas, nuevamente, por necesidades fiscales. Sólo en México, donde el consumo de estas bebidas es extraordinario y afronta costos sanitarios descomunales por ello (10), el incremento de impuestos de 2014 parece haber sido verdaderamente motivado por razones de salud pública, debido a que se debatió y analizó en el contexto de una serie de medidas complementarias (restricciones a la publicidad, etiquetado, etc.). Aunque el incremento del impuesto haya sido relativamente moderado (alrededor de $10 \%$ del precio promedio de las bebidas azucaradas), puede haber sido efectivo en disminuir la asequibilidad. En la muestra analizada también coincide que México sea el país con menor tasa de crecimiento de la asequibilidad.

1. Dreyfus PM, Victor M. Effects of Thiamine Deficiency on the Central Nervous System. Am J Clin Nutr. 1961;9(4):414-25.

2. Victor M, Adams RD, Mancall EL. A restricted form of cerebellar cortical degeneration occurring in alcoholic patients. AMA Arch Neurol. 1959;1(6):579-688.

3. Smyth A, Teo KK, Rangarajan S, O'Donnell M, Zhang X, Rana P, et al. Alcohol consumption and cardiovascular disease, cancer, injury, admission to hospital, and mortality: a prospective cohort study. Lancet. 2015;386(10007):1945-54.

4. Grant BF, Dawson DA. Age at onset of alcohol use and its association with DSM-IV alcohol abuse and dependence: results from the National Longitudinal Alcohol Epidemiologic Survey. J Subst Abuse. 1997;9(1):103-10.

5. Gawryszewski VP, Monteiro MG. Mortality from diseases, conditions and injuries where alcohol is a necessary cause in the Americas, 2007-09. Addiction. 2014; 109(4):570-7.

6. Reardon DC, Coleman PK, Cougle JR. Substance Use Associated with
Si verdaderamente se desea disminuir su consumo, es recomendable que los países de la Región avancen decididamente en la implementación de estos impuestos con un criterio sanitario. Tal como se conoce en el caso del tabaco, los impuestos específicos (un monto por la unidad de medida correspondiente -volumen, cantidad de nutrientes críticos, etc. -) son los más efectivos para modificar comportamientos (34).

En relación con las bebidas azucaradas, sería deseable que el impuesto tuviera relación con la cantidad de nutrientes críticos que poseen los productos (por ejemplo, azúcares añadidos). Podría pensarse, entonces, en gravar con impuestos las bebidas que tengan un componente específico (monto monetario por gramo de azúcares añadido), de forma que se penalice a las bebidas más intensivas en dichos componentes y se incentive a los consumidores a sustituirlas por alternativas más saludables, al tiempo que se fomenta su reformulación por parte de los productores. Ningún país de los analizados tiene este tipo de impuesto.

Para las bebidas alcohólicas podría pensarse en un impuesto específico por gramo de alcohol puro de forma tal que los consumidores se vean incentivados a reducir el consumo de bebidas con mayor graduación, a sustituirlas por las de

\section{REFERENCIAS}

Unintended Pregnancy Outcomes in the National Longitudinal Survey of Youth. Am J Drug Alcohol Abuse. 2004;30(2): 369-83.

7. Stueve A, O'Donnell LN. Early alcohol initiation and subsequent sexual and alcohol risk behaviors among urban youths. Am J Public Health. 2005;95(5):887-93.

8. Shield K, Monteiro M, Roerecke M, Smith $\mathrm{B}$, Rehm J. Alcohol consumption and burden of disease in the Americas in 2012: implications for alcohol policy. Rev Panam Salud Publica. 2015;38(6):442-9.

9. Vartanian LR, Schwartz MB, Brownell KD. Effects of soft drink consumption on nutrition and health: a systematic review and meta-analysis. Am J Public Health. 2007; 97(4):667-75.

10. Singh GM, Micha R, Khatibzadeh S, Lim S, Ezzati M, Mozaffarian D. Estimated Global, Regional, and National Disease Burdens Related to Sugar-Sweetened Beverage Consumption in 2010. Circulation. 2015; 132(8):639-66.

11. Euromonitor International. Passport data 2017. Disponible en: https://www.portal. menor graduación alcohólica o a dejar de consumirlas completamente. Sólo tres países de los considerados aquí poseen este tipo de impuestos: Colombia, Ecuador y República Dominicana.

El funcionamiento del instrumento tributario depende del objetivo para el que se impone. En el caso que nos interesa, el objetivo debe ser primordialmente el de disminuir el consumo de sustancias nocivas para la salud. Su diseño resulta fundamental y debe estar alineado con este objetivo.

Agradecimiento. Los autores agradecen los valiosos comentarios de Rosa Sandoval, Itziar Belaustiguigoitia, Luis Galicia y Alejandra Guira. Los errores y omisiones que puedan existir son de exclusiva responsabilidad de los autores.

Financiación. Guillermo Paraje agradece el financiamiento recibido de la Organización Panamericana de la Salud para la realización de este estudio.

Conflictos de interés. Los autores declaran no tener conflictos de interés.

Declaración. Las opiniones expresadas en este manuscrito son responsabilidad del autor y no reflejan necesariamente los criterios ni la política de la RPSP/PAJPH y/o de la OPS euromonitor.com/portal/magazine/homemain Acceso el 15 de septiembre de 2017.

12. The World Bank. The World Bank Database. Washington, DC: The World Bank; 2017. Disponible en: http://data.worldbank. org/ Acceso el 21 de mayo de 2017.

13. Rabinovich L, Brutscher $\mathrm{P}$, de Vries $\mathrm{H}$, Tiessen J, Clift J, Reding A. The affordability of alcoholic beverages in the European Union: Understanding the link between alcohol affordability, consumption and harms. Santa Monica, CA: RAND Corporation; 2009.

14. Nelson JP. Alcohol Affordability and Alcohol Demand: Cross-Country Trends and Panel Data Estimates, 1975 to 2008. Alcoholism: Clin Exper Res. 2014;38(4): 1167-75.

15. Blecher EH, van Walbeek CP. An international analysis of cigarette affordability. Tobacco Control. 2004;13(4):339-46.

16. Araya D. El impacto del precio en el consumo de bebidas alcohólicas en Chile. Santoago de Chile: Universidad Adolfo Ibañez; 2016. 
17. Colchero MA, Salgado JC, Unar-Munguía M, Hernández-Ávila M, RiveraDommarco JA. Price elasticity of the demand for sugar sweetened beverages and soft drinks in Mexico. Econ Human Biol. 2015;19(1):129-37.

18. Claro RM, Levy RB, Popkin BM, Monteiro CA. Sugar-Sweetened Beverage Taxes in Brazil. Am J Public Health. 2012;102(1): 178-83.

19. Guerrero-López CM, Unar-Munguía M, Colchero MA. Price elasticity of the demand for soft drinks, other sugar-sweetened beverages and energy dense food in Chile. BMC Public Health. 2017;17(1):180.

20. Nakhimovsky SS, Feigl AB, Avila C, O'Sullivan G, Macgregor-Skinner E, Spranca M. Taxes on Sugar-Sweetened Beverages to Reduce Overweight and Obesity in Middle-Income Countries: A Systematic Review. PloS one. 2016; 11(9):e0163358.

21. Paraje G. The Effect of Price and SocioEconomic Level on the Consumption of Sugar-Sweetened Beverages (SSB): The Case of Ecuador. PloS one. 2016;11(3): e0152260.

22. Gallet CA. The demand for alcohol: a meta-analysis of elasticities. Austral J Agric Resource Econ. 2007;51(2):121-35.

23. Thow AM, Downs S, Jan S. A systematic review of the effectiveness of food taxes and subsidies to improve diets: Understanding the recent evidence. Nutr Rev. 2014;72(9):551-65.

24. International Monetary Fund. Statement by the IMF Executive Board on Argentina [press release]. Washington, DC: IMF; 2013.

25. Guindon GE, Tobin S, Yach D. Trends and affordability of cigarette prices: ample room for tax increases and related health gains. Tobacco Control. 2002; 11(1):35.

26. Blecher EH, van Walbeek CP. Cigarette affordability trends: an update and some methodological comments. Tobacco Control. 2009;18(3):167-75.

27. Hamilton J. Time Series Analysis. Princeton: Princeton University Press; 1994.

28. Newey WK, West KD. A Simple, Positive Semi-Definite, Heteroskedasticity and Autocorrelation Consistent Covariance Matrix. Econometrica. 1987;55(3):703-8.

29. Newey WK, West KD. Automatic Lag Selection in Covariance Matrix Estimation. Rev Econ Studies. 1994;61(4):631-53.

30. Pincheira P, Medel C. Forecasting Inflation with a Simple and Accurate Benchmark: The Case of the US and a Set of Inflation Targeting Countries. Czech J Econ Finance (Finance a uver). 2015; 65(1):2-29.
31. Colchero MA, Popkin BM, Rivera JA, Ng $\mathrm{SW}$. Beverage purchases from stores in Mexico under the excise tax on sugar sweetened beverages: observational study. Br Med J. 2016;352.

32. Silver LD, Ng SW, Ryan-Ibarra S, Taillie LS, Induni M, Miles DR, et al. Changes in prices, sales, consumer spending, and beverage consumption one year after a tax on sugar-sweetened beverages in Berkeley, California, US: A before-andafter study. PLoS medicine. 2017;14(4): e1002283.

33. Elder RW, Lawrence B, Ferguson A, Naimi TS, Brewer RD, Chattopadhyay SK, et al. The Effectiveness of Tax Policy Interventions for Reducing Excessive Alcohol Consumption and Related Harms. Am J Prev Med. 2010;38(2):217-29.

34. International Agency for Research on Cancer. Effectiveness of Tax and Price Policies for Tobacco Control. Lyon: The World Health Organization; 2011.

Manuscrito recibido el 3 de octubre de 2017. Aceptado para publicación, tras revisión, el 4 de enero de 2017.
ABSTRACT

\section{Affordability of beer and soft drinks in 15 Latin American countries}

Keywords
Objective. The objective of this study was to look at trends in the affordability of beer and soft drinks in 15 Latin American countries.

Methods. The data correspond to government statistics pertaining to price indices for beer and soft drinks, the consumer price index, and the nominal wage index based on monthly frequency. The methods involved the performance of an econometric analysis, using time series, to measure the expected rate of increase in the absolute affordability indicator (using nominal prices) or the relative affordability indicator (using general prices) for soft drinks and beers.

Results. In nine of the 15 countries analyzed, the affordability of soft drinks or beer (whether absolute or relative) has shown a statistically significant increase. In the case of soft drinks, absolute affordability increased in five countries (Chile, Colombia, Costa Rica, Ecuador, and Uruguay) and decreased in Mexico. In the case of beer, it increased in Colombia and Ecuador, remained unchanged in Argentina, Brazil, Chile, Costa Rica, and Uruguay, and dropped in Mexico.

Conclusions. Although most countries levy taxes on beer and soft drinks, the effect of such taxes on price has not been enough to reduce the affordability of these products in all countries in the sample. Taxes should be modified to make these beverages less affordable and have an impact on their consumption.

Health Economics; Alcoholic drinks; Soft drinks; Latin America. 
RESUMO Objetivos. Analisar a evolução da acessibilidade a cervejas e refrigerantes em 15 países da América Latina.

\section{Acessibilidade a cervejas e refrigerantes em 15 países da América Latina}

Métodos. Os dados representam estatísticas oficiais dos índices de preços de cervejas e refrigerantes, índice de preços ao consumidor e índice mensal do salário nominal. A metodologia do estudo foi uma análise econométrica com séries temporais para avaliar a taxa esperada de crescimento do indicador de acessibilidade absoluta (com base no salário nominal) ou relativa (com base nos preços gerais) de cervejas e refrigerantes.

Resultados. Houve um aumento significativo da acessibilidade (absoluta ou relativa) a cervejas ou refrigerantes em 9 dos 15 países analisados. Com relação aos refrigerantes, houve um aumento da acessibilidade absoluta em cinco países (Chile, Colômbia, Costa Rica, Equador e Uruguai), com uma redução observada no México. Com relação às cervejas, houve um aumento da acessibilidade na Colômbia e Equador, não se observou mudança na Argentina, Brasil, Chile, Costa Rica e Uruguai e ocorreu uma redução no México.

Conclusões. Embora exista tributação para cervejas e refrigerantes na maioria dos países, o efeito nos preços não tem sido suficiente para reduzir a acessibilidade a estes produtos em todos os países da amostra estudada. É preciso reformar a tributação a fim de reduzir a acessibilidade e o consumo destas bebidas.

Palavras-chave Economia da saúde; bebidas alcoólicas; bebidas gasosas; América Latina. 